\title{
Feasibility and limitations of endoscopy in Guyon's canal
}

\author{
Bartłomiej H. Noszczyk, Piotr Zdybek \\ Medical Centre of Postgraduate Education, Warsaw, Poland
}

Videosurgery Miniinv (e-pub, ahead of print) DOI: $10.5114 /$ wiitm.2014.44140

\begin{abstract}
Introduction: This retrospective report summarizes observations from eight operations where the endoscopically assisted approach was used to explore Guyon's canal syndromes of idiopathic aetiology.

Aim: To evaluate the feasibility and limitations of endoscopic Guyon's canal release performed from a distal forearm incision.

Material and methods: Eight charts and video records of eight ulnar tunnel syndrome patients presenting concomitant idiopathic Guyon's canal syndromes were retrospectively reviewed. In all cases endoscopically assisted explorations in Guyon's canals with simultaneous cubital tunnel releases were performed.

Results: In all of the patients the multiple tight bands of the superficial volar carpal ligament forming the canal roof were divided. Some of these bands crossing the nerve in its direct vicinity could have been responsible for the constriction. We were also able to divide the proximal segment of the canal floor. We have observed, however, that the proximal to distal endoscopic dissection jeopardizes the motor branch of the ulnar nerve; therefore, it should not be used to release the pisohamate ligament, or the hypothenar fascia.

Conclusions: Although all of the patients showed improvement, we cannot recommend this method in its current form. We are of the opinion that safe endoscopic Guyon's canal operations may require a different approach.
\end{abstract}

Key words: Guyon's canal syndrome, Guyon's canal release, ulnar nerve, endoscopic exploration, hand surgery, transverse carpal ligament.

\section{Introduction}

The pathophysiology of the cubital tunnel and Guyon's canal syndromes are multifactorial [1, 2]. Cubital tunnel syndrome has, additionally, a multifocal aetiology [3]. More than one compression site may coexist along the proximal and distal course of the ulnar nerve [4]. In order to make an inspection over such a potentially long distance minimally invasive, easier and efficient, surgeons frequently choose an endoscopic approach for cubital tunnel syndrome operations [5]. Moreover, in the short term, the results from endoscopy are probably better than those from the traditional open approach [4].
Although endoscopy enables the release of the fascial bands many centimetres distally from the retrocondylar groove [6], other ulnar neuropathies in the distal forearm and in Guyon's canal are traditionally explored using the open approach. A more extensive open dissection may be especially valuable in some cases of Guyon's canal syndrome (GCS). This is related to fact that the deep (motor) branch of the ulnar nerve, which leaves Guyon's canal, enters between the hypothenar muscles where it is difficult to access along its course [7].

Nevertheless, we are still of the opinion that in cases of simultaneous diagnosis of cubital tunnel syndrome and GCS, an endoscopic approach pro-

\section{Address for correspondence}

Bartłomiej H. Noszczyk MD, PhD, Medical Centre of Postgraduate Education, 231 Czerniakowska St, 00-416 Warsaw, Poland,

phone: +48 602795 165, fax: +48 2258413 46, e-mail: noszczyk@melilot.pl 
vides a unique possibility to explore the ulnar nerve in the forearm and in the canal without the need of an open incision. The GCS pathology is often caused by ganglions and other organic lesions [8]. In many cases, however, the aetiology of GCS is idiopathic. In such cases the cause of the compression can only be found during exploration of Guyon's canal [9].

We present a retrospective review of eight cubital tunnel syndrome patients presenting concomitant idiopathic GCS, where endoscopically assisted explorations in Guyon's canals and simultaneous cubital tunnel releases were effective.

\section{Aim}

The goal of the study was to summarize our observations and evaluate the feasibility of endoscopic Guyon's canal release.

\section{Material and methods}

We retrospectively reviewed the eight charts and video records of the patients ( 6 women and 2 men) enrolled and operated on in our department between 2010 and 2013. The surgery in Guyon's canals was performed as an additional procedure along with the cubital tunnel release. We included only the charts of those patients diagnosed with cubital tunnel syndrome and simultaneous idiopathic GCS. Preoperatively all of the patients had their diagnoses confirmed with nerve conduction studies and ultrasonography performed in order to exclude any or-

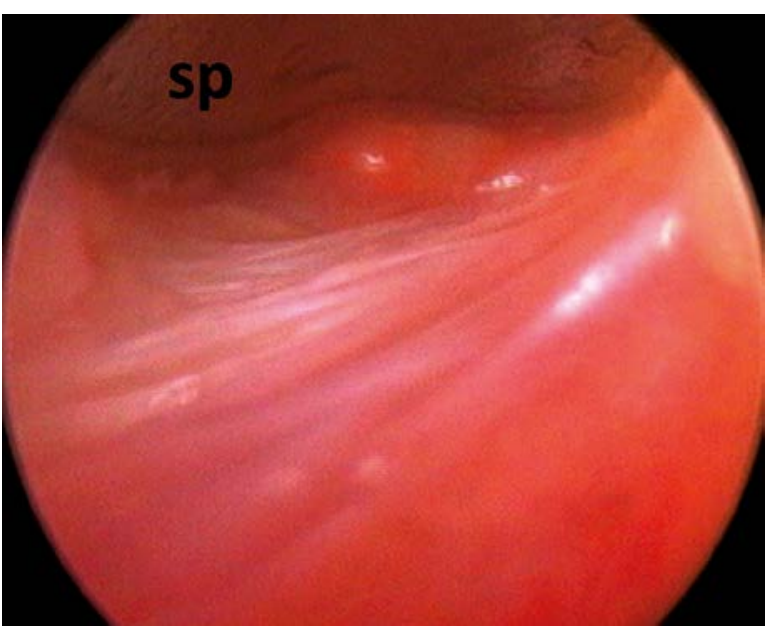

Photo 1. Antebrachial fascia fibres forming the roof of Guyon's canal. In the upper part of the picture, the spatula (sp) on the distal end of the endoscope sheath is visible ganic causes of GCS. The electrophysiological studies were performed by two experienced neurologists. The parameters considered as normal, above which GCS was confirmed, were: the distal motor latency measured from the wrist to the first dorsal interosseous ( $3.3 \mathrm{~ms}$ ) and from the wrist to the abductor digiti minimi ( $2.8 \mathrm{~ms})$.

The sensory function evaluated preoperatively, and then 3 months postoperatively, was examined as a two-point discrimination (2PD) on the ulnar side of the small finger and on the radial and $u l-$ nar side of the ring finger. The carpal tunnel questionnaires (CTQ), with separate fields for a symptom severity score (SSS) and a function severity score (FSS), were administered preoperatively and then 3 months postoperatively to all of the patients. Results of questionnaires were expressed as mean and standard deviation. The study protocol was accepted by the University Bioethical Committee.

\section{Statistical analysis}

Differences were estimated by paired $t$-test. Values of $p$ less than 0.003 were considered significant.

\section{Results}

\section{Observations from surgery}

All of the operations were performed by the senior author (BN). Before the incision the position of the pisiform was marked on the skin with ink. If the hamate was palpable, it was also marked. The skin was incised on the distal forearm with a $1 \mathrm{~cm}$ cut, perpendicularly to the proximal wrist crease and along the radial side of the flexor carpi ulnaris tendon. The ulnar artery was found under the fascia and retracted to provide safe access to the nerve. Under direct control the ulnar nerve was followed distally to the level of the entrance into Guyon's canal. It was possible to lift the skin with a retractor at this time in order to perceive the proximal edge of the canal. A $4 \mathrm{~mm} 0^{\circ}$ endoscope, protected by an irrigation sheath with a distal beak for restraining tissue (Karl Storz GmbH, Tuttlingen, Germany) was inserted into the subcutaneous fat. The sheath's beak forms a narrow spatula which can be seen in the monitor (Photo 1) and palpated under the skin. The exact place of dissection may therefore be controlled through the skin.

When the endoscope was inserted into the subcutaneous tissue, the canal roof, which consists of 
antebrachial fascia (Photo 1) and the irregular fibres of the palmaris brevis muscle, becomes visible under the skin. Below the nerve the proximal part of the floor of Guyon's canal, which is formed by the transverse carpal ligament, could be perceived. The fibres forming the roof were incised with delicate scissors. The proximal part of the canal floor can also be incised when the nerve is retracted to the side. After fascia release the endoscope was inserted contiguously to the nerve and in its direct vicinity. Further dissection at this level enabled the incision of the fibres wrapping the nerve surface (Photo 2). In 5 patients a number of these fibres formed tight cords immobilizing the nerve on the canal floor (Photos $3 \mathrm{~A}, \mathrm{~B}$ ). They were identified and released over a distance of a few millimetres distally from the pisiform.

At the level of the pisiform the distal edge of the pisohamate ligament forming the distal part of the canal floor was, on occasion, visible. Its division was not performed. To facilitate its identification the endoscope could be lifted under the skin. In two patients such traction created the sling in which the nerve seemed to be suspended (Photo 4). Distally from the pisohamate ligament the leading edge of the hypothenar, where the deep motor branch separates radially from the nerve, was occasionally identified.

Subsequently the ulnar nerve at the distal third of the forearm was explored. The endoscope was introduced proximally, from the same skin incision on the distal forearm. The deep fascia overlying the nerve was incised $6-8 \mathrm{~cm}$ proximally. The endoscope

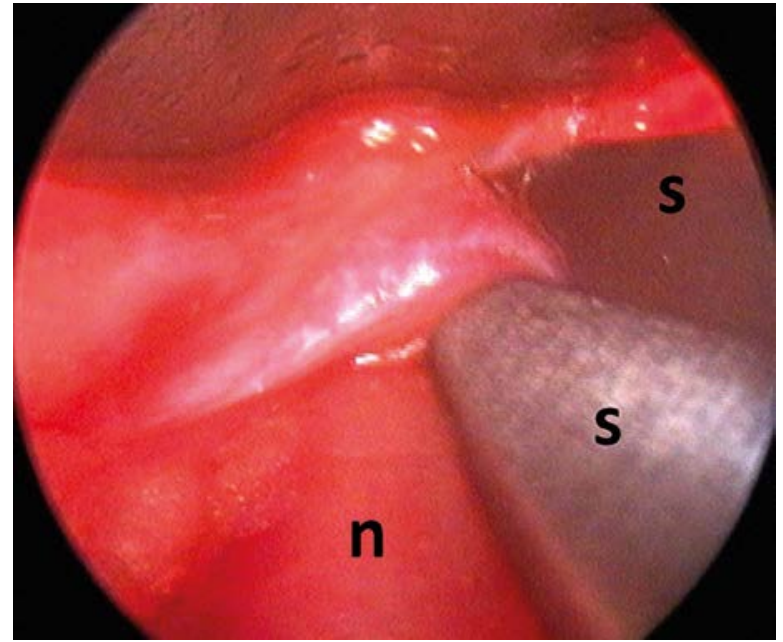

Photo 2. The thick fascial fibre crossing the ulnar nerve is incised with scissors

$n$-the ulnar nerve, $s$-scissors

was advanced in the vicinity and superficially to the nerve. All the bands crossing the nerve in the subcutaneous tissue and proximally subjacent to the flexor carpi ulnaris were identified and divided. Although further proximal advancement was possible, it was not performed.

\section{Clinical observations}

Preoperatively all of the patients complained about paraesthesia over the ulnar side of their hands. They described their symptoms as pain, or a painful tingling accompanying efforts made, or
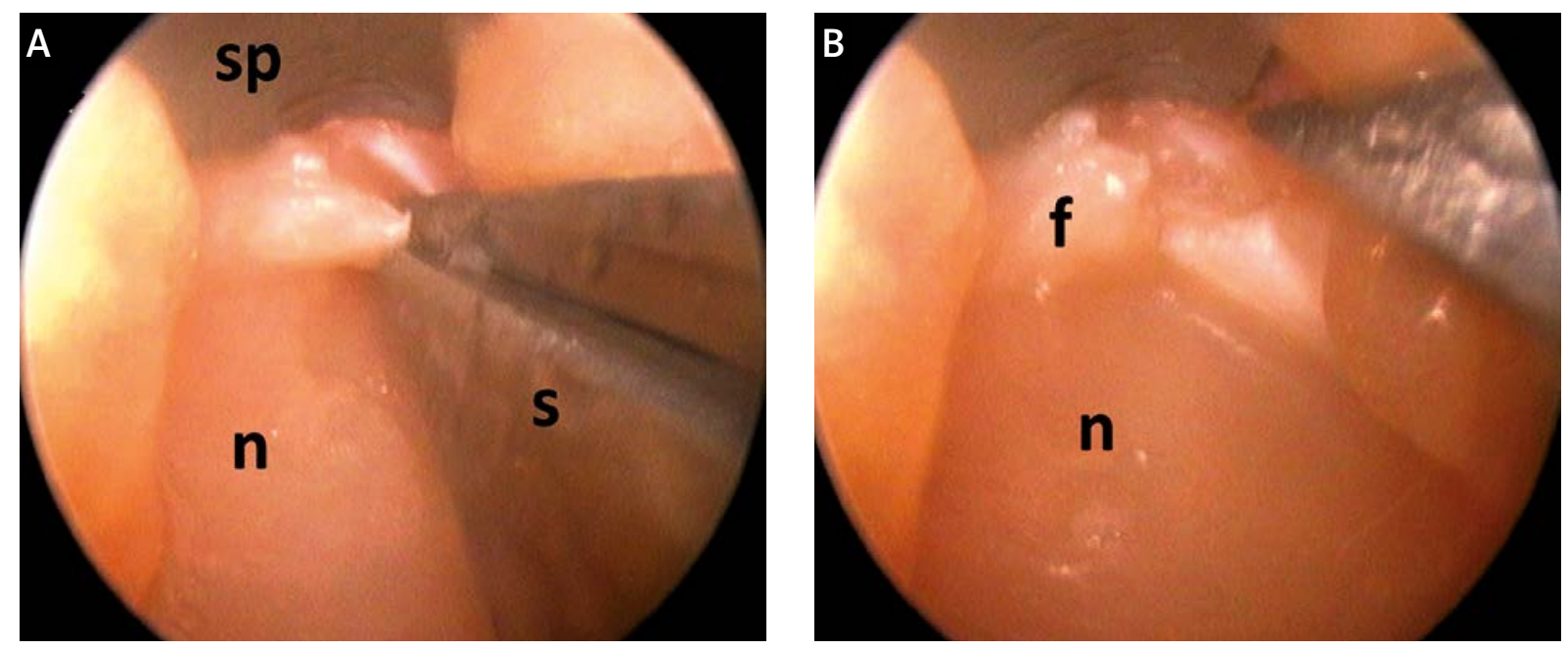

Photo 3. A - Tight fibres constricting the ulnar nerve, before division. B - Fibres constricting the ulnar nerve after partial division

$n$ - the ulnar nerve, s-scissors, sp-endoscope spatula, $f$ - incised fibres 


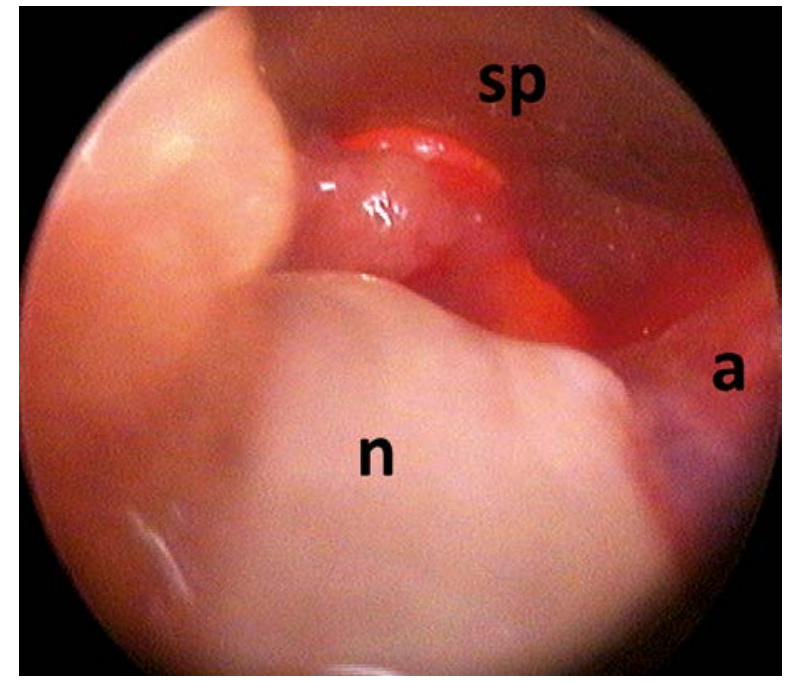

Photo 4. The ulnar nerve seems to be suspended over the pisohamate ligament. The ulnar artery is visible on the right side of the nerve

$n$ - the ulnar nerve, a - ulnar artery, sp - endoscope spatula

other activities. All of the patients complained, to various degrees, of hand weakness, but none had visible muscle atrophy.

Postoperatively all of the patients observed sensory improvement by means of 2PD decrease. Severity of symptoms (SSS) decreased significantly one month after the operation (Figure $1 \mathrm{~A}$ ). Function impairment at 1 month improved only insignificantly. At 3 months FSS decreased significantly, reflecting functional improvement (Figure $1 \mathrm{~B}$ ). At this time all of the patients had observed an improvement in the strength of their treated hands.

\section{Discussion}

In all our patients the endoscopy in Guyon's canals was planned primarily for exploration. On no occasion did we attempt to release the canal, compromising the safety of the neurovascular bundles. In all of the patients the multiple bands of the superficial volar carpal ligament forming the roof of Guyon's canal were observed in the entrance to Guyon's canal. Such bands were often interlaced with fat and distributed in groups (Photo 2). They crossed the nerve without direct contact. However, distally further we identified very firm and tight bands which crossed the nerve in its immediate vicinity (Photos $3 \mathrm{~A}, \mathrm{~B})$. We are of the opinion that these fibres could be responsible for the constriction. We were also able to divide the proximal segment of the canal floor. This manoeuvre is easy proximally, but seems to be dangerous when the dissection is performed in the distal part of the canal. For this reason we
A

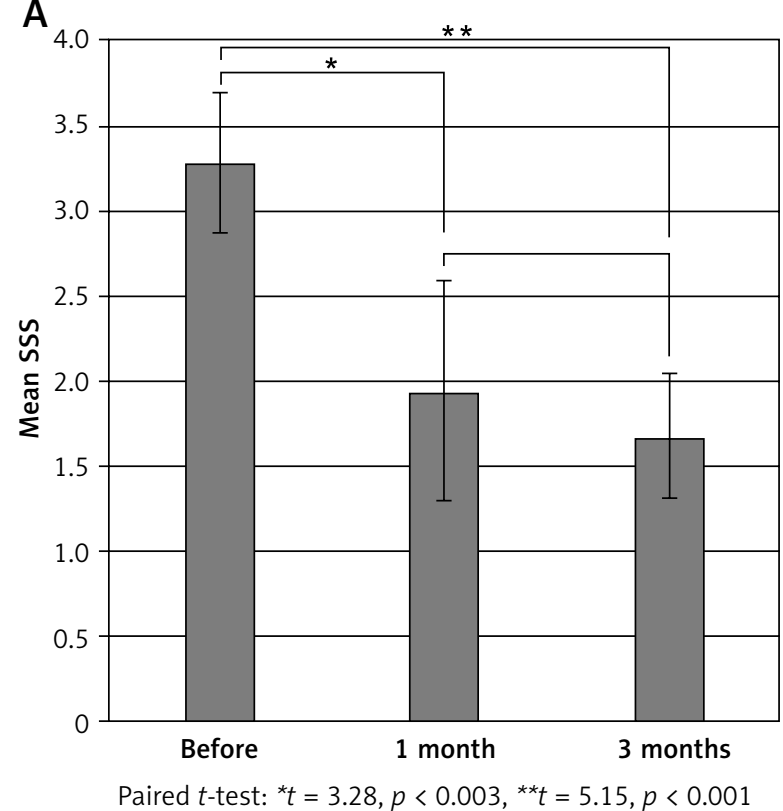

B

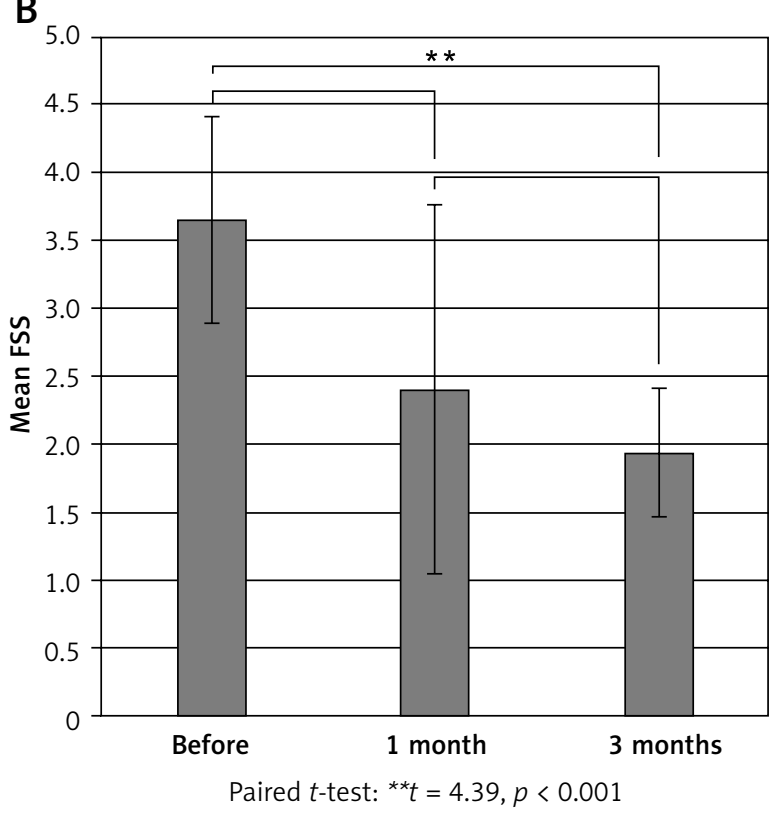

Figure 1. A - Significant decrease of SSS at 1 and at 3 months postoperatively. B - Significant decrease of FSS may be observed only after 3 months postoperatively 
were unwilling to attempt incising the pisohamate ligament. The leading edge of the hypothenar was also not incised.

Despite Okutsu's first report on the use of an endoscope for the management of carpal tunnel syndrome some 20 years ago [10], new applications of endoscopy for constriction syndromes of the upper extremity are still readily accepted by surgeons and appreciated by patients. Only recently has it been successfully employed for radial nerve decompression [11] and for pronator syndrome [12]. Its use for Guyon's canal has not, however, been described. This is probably related to the fact that it jeopardizes the motor branch of the ulnar nerve, and is inefficient to follow its course between the hypothenar muscles.

The idea of using it, with a conscious regard as to the risks involved, may, perhaps, be justified in patients with concomitant cubital tunnel syndrome and GCS. In these patients a short additional incision in the distal forearm may facilitate endoscopic access to the entire ulnar nerve, including its small segment within the canal. We have review outcomes of eight endoscopically assisted operations of GCS, and have confirmed that they are effective. Even though the release of the motor branch was not performed in any of the cases, all of our patients observed improvement. However, our study has obvious limitations, and too many doubts exist before being able to recommend this technique in its present form.

The limited number of patients and the retrospective character of our observations makes it casuistic. Furthermore, all of our patients had concomitant cubital tunnel syndrome and GCS. The CTQ questionnaire was recently recommended [13] for a postoperative assessment of isolated cubital tunnel syndrome cases. The patient-reported outcomes were found to be more responsive indicators of improvement than grip strength and sensory testing [14]. However, according to recent observations, the GCS patients usually manifest motor rather than sensory deficits [15] We did not evaluate the grip strength in our patients. Moreover, the CTQ, which was originally developed for the carpal tunnel, may not be similarly responsive in GCS patients. Our methods of assessment may therefore be adequate for isolated cubital tunnel syndrome cases. GCS symptoms persistence could, in this situation, remain unnoticed. Future studies should, perhaps, include the confirmation of postoperative improvement with nerve conduction tests.
All of our patients had idiopathic GCS aetiology. Although the majority of GCS cases in the population are caused by ganglia or anatomical anomalies, $45 \%$ may be idiopathic [9]. Murata et al. observed that many idiopathic cases had compressions within the motor branch of the ulnar nerve. Considering this frequency they advised following the motor branch along its course, by surgical hypothenar exploration [9]. Bachoura and Jacoby also recently recommended releasing the tendinous hypothenar muscles origin [16]. This may be done together with the transverse carpal ligament division. The ligament constitutes the floor of Guyon's tunnel in which the motor branch runs [16]. Both the above-mentioned groups also suggested that the same result for Guyon's canal decompression may be achieved by carpal tunnel release alone $[9,16]$. This observation was also confirmed by pressure measurements of Guyon's canal after carpal tunnel release [17]. The technique described in our study enables the endoscopically assisted release of the fascial roof, as well as part of the ligamentous floor of the canal. We do not believe, however, that it would be efficient in cases of pure motor branch involvement. Unfortunately, recent observations suggest that motor branch pathology may be pathognomonic for GCS [15]. If it is characteristic for every idiopathic GCS case, then indeed the decompression of this branch may require more extensive dissection from an open incision. Future studies, including the possible development of GCS endoscopic decompression from the palmar approach, will perhaps resolve this problem.

\section{Conclusions}

We have described our observations from eight cases of endoscopically assisted explorations of Guyon's canals from distal forearm incisions. In all of the patients the multiple bands forming the roofs and floors of the canals were released. However, this technique proved to be inefficient in decompressing the motor branch of the ulnar nerve. Despite these limitations, all of our patients observed improvement. In part, this was related to the fact that all of the patients had simultaneous ulnar tunnel syndrome, which was also released. Due to the technique limitations and the bias in our results, we cannot recommend this method in its current form. Perhaps some different approaches could be more promising for the endoscopic decompression of Guyon's canal. 


\section{References}

1. Ochi K, Horiuchi Y, Nakamura T, et al. Ulnar nerve strain at the elbow in patients with cubital tunnel syndrome: effect of simple decompression. J Hand Surg Eur 2013; 38: 474-80.

2. Hoogvliet P, Coert JH, Fridén J, Huisstede BM; the European HANDGUIDE group. How to treat Guyon's canal syndrome? Results from the European HANDGUIDE study: a multidisciplinary treatment guideline. Br J Sports Med 2013; 47: 1063-70.

3. Hoffmann R, Siemionow M. The endoscopic management of cubital tunnel syndrome. J Hand Surg Br 2006; 31: 23-9.

4. Dützmann S, Martin KD, Sobottka S, et al. Open vs retractor-endoscopic in situ decompression of the ulnar nerve in cubital tunnel syndrome: a retrospective cohort study. Neurosurgery 2013; 72: 605-16

5. Yoshida A, Okutsu I, Hamanaka I. Endoscopic anatomical nerve observation and minimally invasive management of cubital tunnel syndrome. J Hand Surg Eur 2009; 34: 115-20.

6. Siemionow M, Agaoglu G, Hoffmann R. Anatomic characteris tics of a fascia and its bands overlying the ulnar nerve in the proximal forearm: a cadaver study. J Hand Surg Eur 2007; 32 302-7.

7. Pearce C, Feinberg J, Wolfe SW. Ulnar neuropathy at the wrist. HSS I 2009; 5: 180-3.

8. Polatsch DB, Melone CP Jr, Beldner S, Incorvaia A. Ulnar nerve anatomy. Hand Clin 2007; 23: 283-9.

9. Murata K, Shih JT, Tsai TM. Causes of ulnar tunnel syndrome: a retrospective study of 31 subjects. J Hand Surg Am 2003; 28: 647-51.

10. Okutsu I, Ninomiya S, Takatori Y, Ugawa Y. Endoscopic management of carpal tunnel syndrome. Arthroscopy 1989; 5: 11-8.

11. Leclère FM, Bignion D, Franz T, et al. Endoscopically assisted nerve decompression of rare nerve compression syndromes at the upper extremity. Arch Orthop Trauma Surg 2013; 133: 575-82.

12. Lee AK, Khorsandi M, Nurbhai N, et al. Endoscopically assisted decompression for pronator syndrome. J Hand Surg Am 2012; 37: 1173-9.

13. Song JW, Waljee JF, Burns PB, et al. Surgery for the Ulnar Nerve (SUN) Study Group. An outcome study for ulnar neuropathy at the elbow: a multicenter study by the surgery for ulnar nerve (SUN) study group. Neurosurgery 2013; 72: 971-81.

14. Giladi AM, Gaston RG, Haase SC, et al. Surgery of the Ulnar Nerve Study Group. Trend of recovery after simple decompression for treatment of ulnar neuropathy at the elbow. Plast Reconstr Surg 2013; 131: 563e-73e.

15. Kollmer J, Bäumer P, Milford D, et al. T2-signal of ulnar nerve branches at the wrist in Guyon's canal syndrome. PLoS One 2012; 7: e47295.

16. Bachoura A, Jacoby SM. Ulnar tunnel syndrome. Orthop Clin North Am 2012; 43: 467-74.

17. Okutsu I, Hamanaka I, Yoshida A. Pre- and postoperative Guyon's canal pressure change in endoscopic carpal tunnel release: correlation with transient postoperative Guyon's canal syndrome. J Hand Surg Eur 2009; 34: 208-11.

Received: 3.11.2013, accepted: 28.02.2014. 